Site selective growth of heteroepitaxial diamond nanoislands containing single SiV centers

Carsten Arend, Patrick Appel, Jonas Nils Becker, Marcel Schmidt, Martin Fischer, Stefan Gsell, Matthias Schreck, Christoph Becher, Patrick Maletinsky, and Elke Neu

Citation: Appl. Phys. Lett. 108, 063111 (2016); doi: 10.1063/1.4941804

View online: http://dx.doi.org/10.1063/1.4941804

View Table of Contents: http://aip.scitation.org/toc/apl/108/6

Published by the American Institute of Physics 


\title{
Site selective growth of heteroepitaxial diamond nanoislands containing single SiV centers
}

\author{
Carsten Arend, ${ }^{1}$ Patrick Appel, ${ }^{2}$ Jonas Nils Becker, ${ }^{1}$ Marcel Schmidt, ${ }^{1}$ Martin Fischer, ${ }^{3}$ \\ Stefan Gsell, ${ }^{3}$ Matthias Schreck, ${ }^{3}$ Christoph Becher, ${ }^{1}$ Patrick Maletinsky, ${ }^{2}$ and Elke Neu ${ }^{1,2}$ \\ ${ }^{1}$ FR 7.2 (Experimentalphysik), Universität des Saarlandes, Campus E2.6, D-66123 Saarbrücken, Germany \\ ${ }^{2}$ Departement Physik, Universität Basel, Klingelbergstrasse 82, CH-4056 Basel, Switzerland \\ ${ }^{3}$ Lehrstuhl für Experimentalphysik IV, Universität Augsburg, D-86135 Augsburg, Germany
}

(Received 6 November 2015; accepted 30 January 2016; published online 11 February 2016)

\begin{abstract}
We demonstrate the controlled preparation of heteroepitaxial diamond nano- and microstructures on silicon wafer based iridium films as hosts for single color centers. Our approach uses electron beam lithography followed by reactive ion etching to pattern the carbon layer formed by bias enhanced nucleation on the iridium surface. In the subsequent chemical vapor deposition process, the patterned areas evolve into regular arrays of (001) oriented diamond nano-islands with diameters of $<500 \mathrm{~nm}$ and a height of $\approx 60 \mathrm{~nm}$. In the islands, we identify single $\mathrm{SiV}$ color centers with narrow zero phonon lines down to $1 \mathrm{~nm}$ at room temperature. (C) 2016 AIP Publishing LLC.

[http://dx.doi.org/10.1063/1.4941804]
\end{abstract}

Color centers in diamond are being extensively investigated as stable, room temperature single photon emitters ${ }^{1}$ simultaneously hosting highly controllable electronic spin systems. They are promising candidates for quantum information processing architectures (single photon sources and spin qubits) and as quantum sensors (e.g., for magnetic fields ${ }^{2}$ and optical near fields ${ }^{3}$ ). For all these applications, an efficient collection of fluorescence light emitted by color centers is crucial. The high refractive index of diamond $(n=2.4)$ here is an ambivalent property: on one hand, it renders light extraction from bulk material highly challenging due to total internal reflection. On the other hand, it enables controlling the emission properties of color centers using versatile diamond photonic structures like waveguides and nanocavities. ${ }^{4}$ Recent work on color centers in diamond often uses top-down fabricated single-crystal diamond nano/microstructures enabling efficient light collection. Top-down nanofabrication creates many photonic structures, e.g., nanopillars, in regular arrays in which color centers can be straightforwardly identified and (re-)addressed. ${ }^{5}$ However, diamond nanofabrication requires sophisticated, non-standard procedures, e.g., for plasma etching, that are challenging due to the chemical inertness of diamond. Avoiding diamond nanofabrication, ${ }^{5}$ enhanced outcoupling of light is alternatively obtained using nanodiamonds (NDs) combined with non-diamond photonic structures. ${ }^{4}$ However, color centers in NDs may suffer from unstable fluorescence and short spin coherence times. Random spatial placement and orientation of NDs, e.g., resulting from spincoating deposition, renders identifying and re-addressing suitable color centers challenging. In this paper, we introduce an approach to unite the advantages of ND based systems and top-down fabrication of photonic structures, i.e., controlled growth of regular arrays of heteroepitaxial diamond nano- and microstructures on iridium (Ir).

In previous work, regular arrays of diamond nanostructures have been obtained by chemical vapor deposition (CVD) on diamond substrates through openings in a mask. ${ }^{6-9}$ However, etching of the mask material in the CVD plasma led to the formation of high densities of color centers. ${ }^{6,7}$ Thus, this method can so far not be considered as an approach capable of high purity diamond growth for single color center applications. Moreover, for this system a significant fraction of color center fluorescence is emitted into the growth substrate (diamond). ${ }^{6,7}$ Using ND seeding, the growth of NDs or ND clusters at pre-defined positions on various substrates has been achieved. ${ }^{10,11}$ However, in all these experiments, identifying single color centers was not feasible.

Here we present an alternative approach to grow regular patterns of diamond micro- and nanostructures for single photon emission. It is based on diamond heteroepitaxy on iridium/yttria-stabilized-zirconia/silicon (Ir/YSZ/Si) substrates. This system has recently been suggested as a highly promising route towards large area single crystal diamond wafers. ${ }^{12}$ Furthermore, the excellent inertness of Ir surfaces towards etching in the CVD growth environment facilitates the synthesis of high purity diamond crystals. Patterned diamond growth has been demonstrated earlier on $\mathrm{Ir} / \mathrm{MgO}$ substrates. ${ }^{13,14}$ However, no investigations of color centers have been performed. In previous work, CVD-NDs grown on ND seeds hosted bright, single silicon vacancy $(\mathrm{SiV})$ centers created in-situ as a result of residual Si incorporation. ${ }^{15}$ The presence of Ir substrates leads to potential collection efficiencies for fluorescence light exceeding 65\% (numerical aperture 0.8 ) via altering the emission characteristics of the color centers. ${ }^{16}$ Heteroepitaxial (001) oriented NDs (nanoislands, NIs) on a randomly structured Ir substrate were used for the spectroscopy of single $\mathrm{SiV}$ centers ${ }^{17}$ which showed $\mathrm{MHz}$ single photon-rates. ${ }^{16}$ However, in all previous work, random spatial placement of NDs or NIs rendered it highly challenging to re-identify promising single $\mathrm{SiV}$ centers and use them for applications.

In this work, we have grown arrays of diamond nanoand microstructures using patterned etching of the carbon layer formed by bias enhanced nucleation (BEN) on Ir. The procedure involves electron beam lithography as well as a dry etch step in an inductively coupled plasma reactive ion 


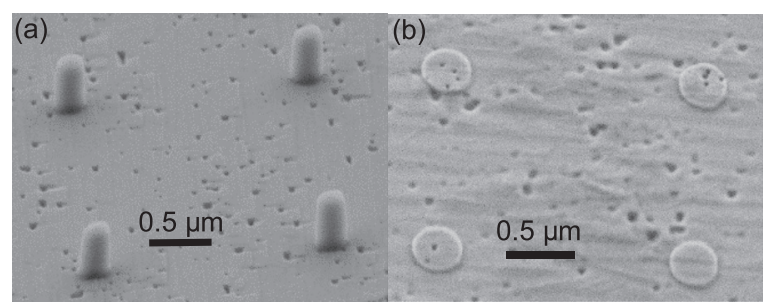

FIG. 1. Scanning electron microscopy (SEM) image under $45^{\circ}$ of (a) example of cylindrical HSQ masks on Ir (b) example of circular nucleation areas defined using $60 \mathrm{~s}$ of ICP-RIE plasma after HSQ mask removal before CVD growth of diamond.

etching system (ICP-RIE) and thus allows us to engineer structures of almost arbitrary, planar geometries. Depending on the growth conditions, we achieve almost perfect selectivity between etched and non-etched areas. Silicon Vacancy $(\mathrm{SiV})$ centers are created in-situ and we observe single centers in diamond nanostructures.

As a growth substrate, we employ an Ir/YSZ/Si multilayer system fabricated on $\mathrm{Si}(001)$ with an off-axis angle of $4^{\circ}$ (for details see Refs. 12 and 18). Heteroepitaxial diamond nucleation is achieved by BEN using a bias voltage of $\approx-300 \mathrm{~V}$ in a gas atmosphere of $3 \% \mathrm{CH}_{4}$ in $\mathrm{H}_{2}$. The typical density of nuclei created in this process is $\approx 3 \times 10^{11} \mathrm{~cm}^{-2}$. To define the patterns, hydrogen silsesquioxane (HSQ) negative electron beam resist (FOX-16, Dow Corning) is spun onto the top Ir layer. We use e-beam lithography $(30 \mathrm{keV})$ to pattern etch-masks into the HSQ layer of $\approx 300-600 \mathrm{~nm}$ thickness [example of HSQ mask see Fig. 1(a)]. After developing the mask, we etch away the BEN layer, which is highly chemically stable but only $\approx 1 \mathrm{~nm}$ thick. ${ }^{19}$ Consequently, the process also etches some of the underlying Ir to ensure full removal of the BEN layer. We use an ICP-RIE (Sentech SI 500) reactor using the following plasma parameters: 50\% Ar and $\mathrm{O}_{2}$, respectively, gas flow $50 \mathrm{sccm}$ each, pressure $0.5 \mathrm{~Pa}$, ICP power $500 \mathrm{~W}$, bias power $200 \mathrm{~W}$. Ar ensures physical etching of BEN layer and Ir, while $\mathrm{O}_{2}$ ensures full oxidation and thus the removal of all carbon components. From scanning electron images of samples etched for $60 \mathrm{~s}$ [see Fig. 1(b)], we estimate an etch rate of $\approx 25 \mathrm{~nm} / \mathrm{min}$ for the Ir layer. Note that this short ICP-RIE etch step does not induce any discernible erosion of the HSQ mask, ${ }^{20}$ indicating that much thinner etch masks might be employed in future work. Efficient etching of the BEN layer is confirmed by the observation (data not shown) that $5 \mathrm{~s}$ of etching removes the BEN layer completely and efficiently suppresses the heteroepitaxial growth of diamond. After etching, we remove the HSQ mask using buffered oxide etch and rinsing in deionized water. As visible in Fig. 1, the Ir layer shows a residual roughness with slight dimples of typically tens of nanometers depth. We note that these dimples, which result from island formation in the nucleation stage of the Ir layer deposition, do not penetrate through the layer and do not negatively influence diamond heteroepitaxy.

On the structured substrates, we grow heteroepitaxial diamond using a microwave-plasma-assisted CVD process (IPLAS reactor with CYRANNUS plasma source). We employ a $\mathrm{CH}_{4} / \mathrm{H}_{2}$ plasma containing $0.5 \%$ or $0.2 \% \mathrm{CH}_{4}$ at a gas pressure of 30 mbar and a microwave power of $2000 \mathrm{~W}$. For $0.5 \%$ $\mathrm{CH}_{4}$, we find a growth rate of $\approx 150 \mathrm{~nm} / \mathrm{h}$, while for $0.2 \% \mathrm{CH}_{4}$ slower growth with only $\approx 70 \mathrm{~nm} / \mathrm{h}$ is observed. $\mathrm{SiV}$ centers are created during CVD growth as a result of etching of the sidewalls of the underlying silicon substrate. Growth at $0.5 \% \mathrm{CH}_{4}$ yields almost perfect selectivity, while for $0.2 \% \mathrm{CH}_{4}$ and longer growth time the selectivity decreases and non-epitaxial crystals appear. Figure 2(a) shows an overview of the pattern grown with $0.5 \% \mathrm{CH}_{4}$ and high selectivity. To test the versatility of the method, we structure not only NIs but also some larger structures like rings, checkerboard patterns and the logo of Basel university. Figure 2(b) shows an array of circular NIs, while Fig. 2(c) shows a close up of a NI with $<500 \mathrm{~nm}$ diameter which is closely resembling the circular shape of the growth area defined in the nanofabrication process. The NIs are especially suitable to observe single color centers due to a small diamond volume and thus higher probability to isolate single color centers. Some missing NIs in the regular pattern arise where the HSQ mask was lost due to insufficient adhesion which might be improved in the future using an adhesion promoter. The ring structures demonstrate the growth of smooth diamond films, which follow the predefined growth areas almost ideally [Fig. 2(d)]. Note some minor diamond growth outside the
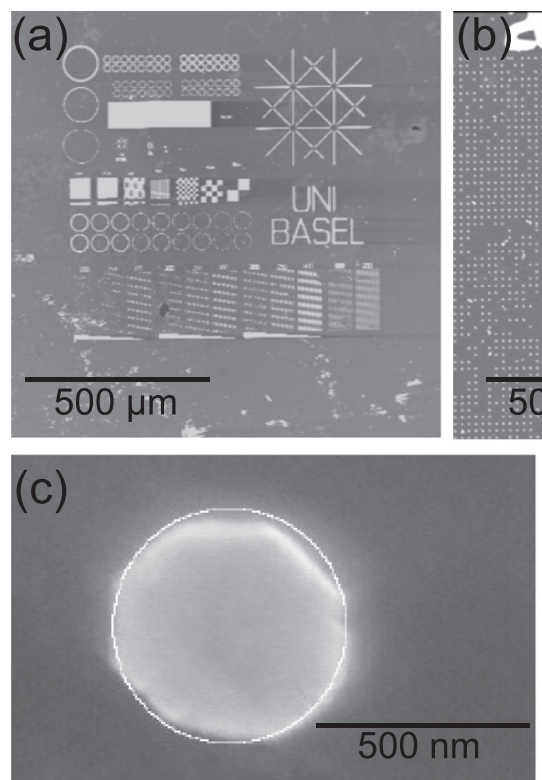
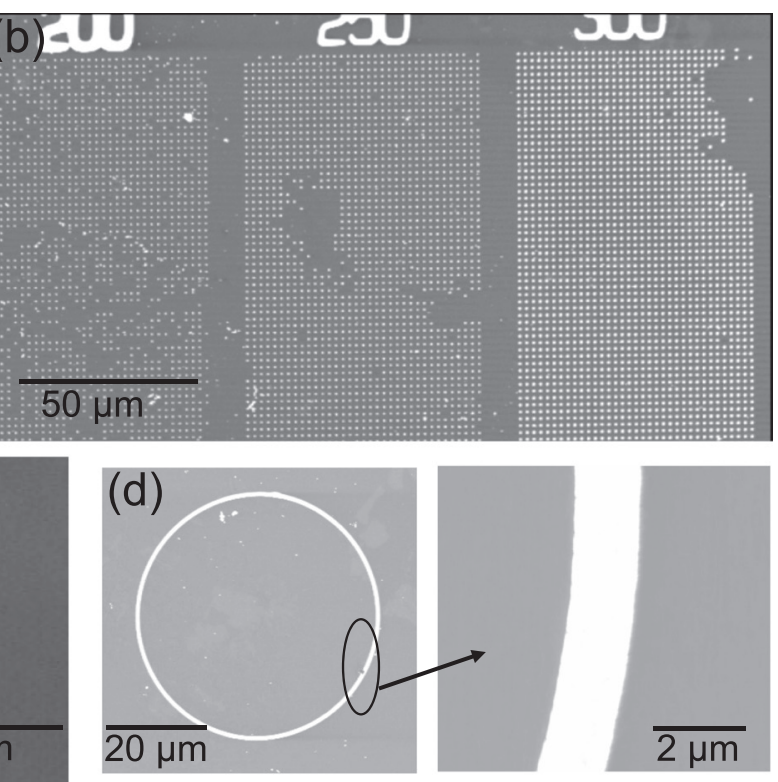

FIG. 2. SEM images (top view) of heteroepitaxial CVD diamond (a) Overview of the pattern (b) NIs with $<500 \mathrm{~nm}$ diameter (c) close up of a diamond NI created by CVD (white circle as guide to the eye) (d) ring structure demonstrating very smooth diamond structures with a diamond thickness of $60 \mathrm{~nm}$. We conclude from additional SEM and AFM images that the top surfaces of the NIs are flat and thus resemble (001) surfaces, whereas the edges of the structures closely follow the pre-defined growth areas. 
pre-defined areas, e.g., inside the diamond ring in Fig. 2(d). This arises most probably from surface contamination that protects the underlying BEN layer during etching.

The samples are analyzed using a homebuilt confocal fluorescence microscope with a Ti: sapphire laser at $700 \mathrm{~nm}$ as excitation source. A $100 \times$ microscope objective with a numerical aperture of 0.8 focuses the laser light onto the sample and collimates the photoluminescence (PL). Excitation laser and PL are separated by a glass beam splitter and dielectric longpass and bandpass filters. A single mode fiber serves as pinhole for the confocal setup and directs the PL to either a Hanbury-Brown Twiss setup (HBT) consisting of two avalanche photodiodes (APD, Perkin Elmer SPCM-AQRH-14) to obtain photon statistics and countrate or to a grating spectrometer (Horiba Jobin Yvon, iHR 550 and Symphony CCD) for spectral analysis. Gratings with 600 (1800) grooves per mm provide a resolution of $0.18(0.06) \mathrm{nm}$. A liquid helium flow cryostat (Janis Research, ST-500LN) can be used for experiments at cryogenic temperatures.

Confocal images obtained recording the PL in a spectral window of 730-750 nm [see Fig. 3] almost perfectly reproduce the diamond pattern fabricated earlier. Figure 3(a) shows a coarse PL image of the pattern in Fig. 2(a), with close up images shown in Figs. 3(b) and 3(c). We observe strong PL from the diamond pattern, while almost no (background) PL is emitted from the etched Ir areas. Examining the spatially well separated NIs [see Fig. 3(d)] and other microstructures on the sample, localized bright spots emerge in the images, while the larger structures predominantly show uniform luminescence intensity see, e.g., Fig. 3(c). Most of the bright spots exhibit spectra with broad ( $>4 \mathrm{~nm}$ ) zero phonon lines (ZPLs) or multiple ZPLs as characteristic for SiV ensembles. Additionally, we find emitters with narrow linewidth SiV ZPLs localized around $740 \mathrm{~nm}$ and linewidths from 1 to $1.8 \mathrm{~nm}$ at room temperature [see Fig. 5 and Ref. 20]. As a rough order of magnitude for the statistics, one out of 100 NIs shows a narrow ZPL with low background, indicating a single $\mathrm{SiV}$ center.
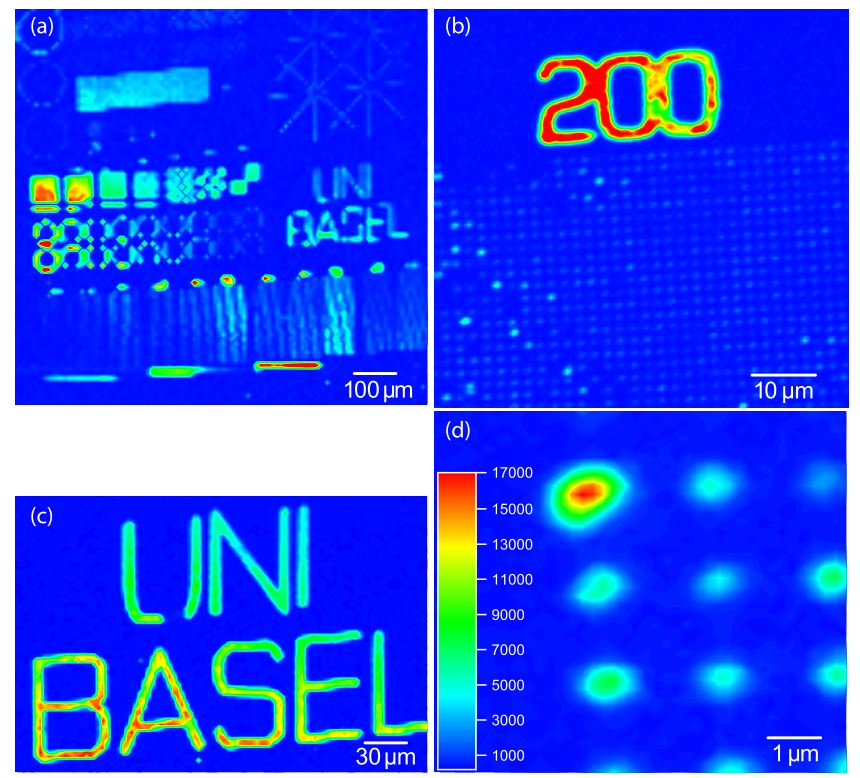

FIG. 3. PL image of (a) the complete pattern of heteroepitaxial CVD diamond (b) NIs with $<500 \mathrm{~nm}$ diameter (c) the Basel university logo (d) spatially well separated NIs at $100 \mu \mathrm{W}$ excitation power.

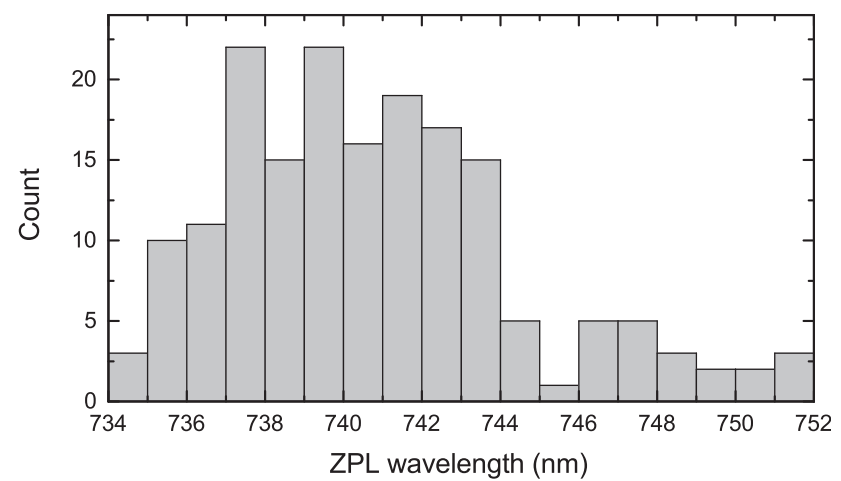

FIG. 4. Histogram of the observed ZPL positions. Spectra with single and several narrow lines were taken into account.

A histogram of the positions of the narrow ZPLs allows us to compare this sample to previously used randomly structured NI samples with regard to strain in the material which is responsible for shifting the ZPL position. ${ }^{17,21}$ Figure 4 shows a spread of the ZPL positions over a range of $18 \mathrm{~nm}$ with a mean value of $740.9 \mathrm{~nm}$ and a standard deviation of $3.9 \mathrm{~nm}$. This distribution is slightly narrower than observed for randomly structured NIs in Ref. 17. Furthermore, the peak of the distribution is closer to the undisturbed SiV ZPL wavelength $(738 \mathrm{~nm})$ and the number of centers with strongly shifted ZPLs $(>744 \mathrm{~nm})$ is reduced. We attribute the shift of the spectral position of the ZPL to stress of the diamond lattice at the position of the color center. A minor fraction can be explained by the biaxial macro stress of about $-0.6 \mathrm{GPa}$ resulting from thermal misfit between diamond and silicon. A major contribution stems from the micro stress of typically several $\mathrm{GPa}$ that can be derived from the line broadening (width of about $15 \mathrm{~cm}^{-1}$ ) in Raman measurements of thin heteroepitaxial diamond layers. ${ }^{22}$ It's formation is characteristic for the merging of the individual grains during the early stage of film growth. However, elastic relaxation can reduce the strain for nanostructures. ${ }^{17,22}$

The observed spread of ZPL positions leads to the conclusion that the sample quality is overall comparable, if not even better, to previously investigated $\mathrm{NDs}^{15}$ and NIs ${ }^{17}$ where bright single photon emission has been demonstrated. We note that the emission rates observed here are significantly lower than previously observed (on the order of several $10000 \mathrm{cps}$ ). The origin of the reduced brightness is currently under investigation.

To identify single SiV centers, we measure the intensity autocorrelation function $g^{(2)}$ of the PL from selected NIs on our sample. Figure 5(b) shows the $g^{(2)}$ function of an emitter
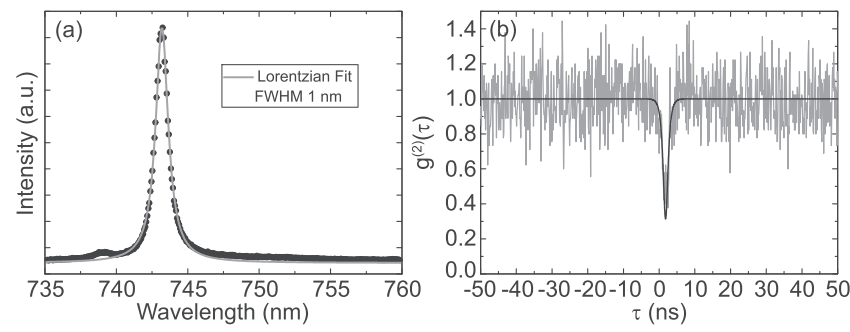

FIG. 5. (a) ZPL of an individual SiV center showing a linewidth of $1 \mathrm{~nm}$ (b) $g^{(2)}$ function of the emitter measured at low excitation power $(25 \mu \mathrm{W})$ fitted with the model for a two level system. 


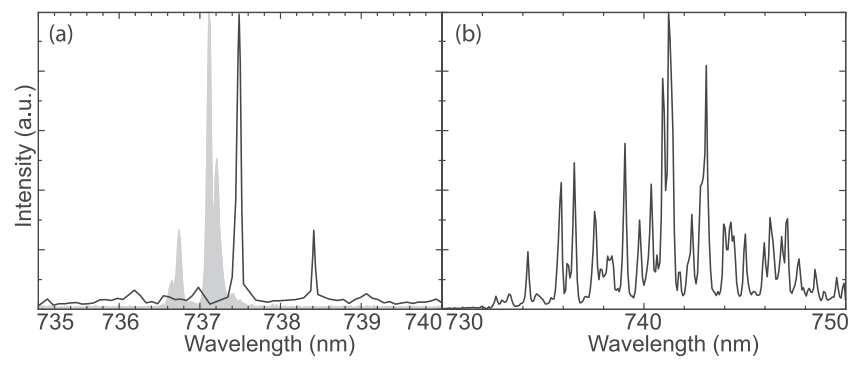

FIG. 6. Spectra at $10 \mathrm{~K}$ showing (a) a dominant line with an underlying ensemble. The gray spectrum has been measured on a low strain CVD sample (compare Ref. 23) (b) a large number of narrow lines.

with a line width of $1 \mathrm{~nm}$ [see Fig. 5(a)]. We observe strong antibunching with $g_{\text {meas }}^{(2)}(0)=0.31$. We fit the data with the $g^{(2)}$ function of a 2 level system ${ }^{1}$ convoluted with the instrument response of our HBT setup which is dominated by the APDs timing jitter. We find excellent agreement with the measured data (residual deviation $\Delta g^{(2)}(0)=0.1$ ). Thus, the observed $g_{\text {meas }}^{(2)}(0)$ value is caused by the timing uncertainty of the APDs in conjunction with the short lifetime of the $\mathrm{SiV}$ center $\left(\tau_{1}=0.7 \mathrm{~ns}\right)$ and we have proven almost pure single photon emission.

For cryogenic temperatures $(<40 \mathrm{~K})$, the $\mathrm{SiV}$ ZPL exhibits a characteristic four line fine structure ${ }^{21}$ in diamond samples with low crystal strain [see gray spectrum in Fig. 6(a)]. At $10 \mathrm{~K}$, we find spectra dominated by single spectrometer resolution-limited lines [see Fig. 6(a)]. These lines most probably represent fine structure lines of single SiV centers modified by crystal strain. Strain can induce a modification of the four line fine structure via changing the thermal population of sublevels and thus quenching some of the lines. ${ }^{21,23}$ Furthermore, we often obtain spectra consisting of many narrow lines, arising from an ensemble of $\mathrm{SiV}$ centers inhomogeneously broadened by crystal strain [see Fig. 6(b)]. Such spectra indicate a comparably high concentration of $\mathrm{SiV}$ centers in our sample, ${ }^{24}$ caused by residual $\mathrm{Si}$ contamination of the CVD chamber. The observation of spectrometer resolution-limited lines indicates the absence of strong spectral diffusion ${ }^{23}$ due to a low influence of other impurities, which is a further sign for high diamond quality.

In summary, we demonstrate the growth of large, regular arrays of (100) oriented heteroepitaxial diamond NIs on Ir substrates. Individual $\mathrm{SiV}$ centers in our nanostructures show narrow ZPLs with a linewidth down to $1 \mathrm{~nm}$. We have thus grown arrays of nanostructures with readdressable emitters for which diamond quality is consistent with previous ND based SiV single photon sources. Further reducing the $\mathrm{Si}$ concentration during growth can lead to a higher yield of single centers. Our material system furthermore allows for a very efficient collection of light emitted by single color centers, thus facilitating their use as single photon sources or sensors. As our approach is able to create almost arbitrary (planar) structures, we envisage the direct growth of photonic structures as, e.g., waveguides ${ }^{25}$ on chip. This bottom up-approach to the fabrication of such nanophotonic components can ease fabrication and broaden the applicability of the devices.

This research has been partially funded by the European Commission's 7. Framework Program (FP7/2007-2013) under Grant Agreement No. 611143 (DIADEMS). E.N. acknowledges funding via the NanoMatFutur program of the German Ministry of Education and Research.

${ }^{1}$ E. Neu and C. Becher, "Diamond-based single-photon sources and their application in quantum key distribution," in Quantum Information Processing with Diamond: Principles and Applications (Woodhead Publishing, Cambridge, 2014) Chap. 6, pp. 127-159.

${ }^{2}$ L. Rondin, J.-P. Tetienne, T. Hingant, J.-F. Roch, P. Maletinsky, and V. Jacques, Rep. Prog. Phys. 77, 056503 (2014).

${ }^{3}$ J. Tisler, T. Oeckinghaus, R. J. Stöhr, R. Kolesov, R. Reuter, F. Reinhard, and J. Wrachtrup, Nano Lett. 13, 3152 (2013).

${ }^{4}$ I. Aharonovich and E. Neu, Adv. Opt. Mater. 2, 911-928 (2014).

${ }^{5}$ T. Babinec, B. Hausmann, M. Khan, Y. Zhang, J. Maze, P. Hemmer, and M. Loncar, Nat. Nanotechnol. 5, 195 (2010).

${ }^{6}$ I. Aharonovich, J. C. Lee, A. P. Magyar, D. O. Bracher, and E. L. Hu, Laser Photonics Rev. 7, L61 (2013).

${ }^{7}$ D. Sovyk, V. Ralchenko, M. Komlenok, A. Khomich, V. Shershulin, V. Vorobyov, I. Vlasov, V. Konov, and A. Akimov, Appl. Phys. A 118, 17 (2015).

${ }^{8}$ H. Masuda, T. Yanagishita, K. Yasui, K. Nishio, I. Yagi, T. N. Rao, and A. Fujishima, Adv. Mater. 13, 247 (2001).

${ }^{9}$ S. Furuyama, K. Tahara, T. Iwasaki, M. Shimizu, J. Yaita, M. Kondo, T. Kodera, and M. Hatano, Appl. Phys. Lett. 107, 163102 (2015).

${ }^{10}$ O. Shimoni, J. Cervenka, T. J. Karle, K. Fox, B. C. Gibson, S. Tomljenovic-Hanic, A. D. Greentree, and S. Prawer, ACS Appl. Mater. Interfaces 6, 8894 (2014).

${ }^{11}$ S. Singh, V. Thomas, D. Martyshkin, V. Kozlovskaya, E. Kharlampieva, and S. A. Catledge, Nanotechnology 25, 045302 (2014).

${ }^{12}$ S. Gsell, T. Bauer, J. Goldfuß, M. Schreck, and B. Stritzker, Appl. Phys. Lett. 84, 4541 (2004).

${ }^{13}$ Y. Ando, J. Kuwabara, K. Suzuki, and A. Sawabe, Diamond Relat. Mater. 13, 1975 (2004).

${ }^{14}$ Y. Ando, T. Kamano, K. Suzuki, and A. Sawabe, Jpn. J. Appl. Phys. 51, 090101 (2012).

${ }^{15}$ E. Neu, D. Steinmetz, J. Riedrich-Möller, S. Gsell, M. Fischer, M. Schreck, and C. Becher, New J. Phys. 13, 025012 (2011).

${ }^{16}$ E. Neu, M. Agio, and C. Becher, Opt. Express 20, 19956 (2012).

${ }^{17}$ E. Neu, M. Fischer, S. Gsell, M. Schreck, and C. Becher, Phys. Rev. B 84, 205211 (2011).

${ }^{18}$ M. Fischer, S. Gsell, M. Schreck, R. Brescia, and B. Stritzker, Diamond Relat. Mater. 17, 1035 (2008).

${ }^{19}$ R. Brescia, M. Schreck, M. Gsell, S. Fischer, and B. Stritzker, Diamond Relat. Mater. 17, 1045 (2008).

${ }^{20}$ See supplementary material at http://dx.doi.org/10.1063/1.4941804 for additional spectra of SiV centers and images of HSQ mask after ICP-RIE etch.

${ }^{21}$ H. Sternschulte, K. Thonke, R. Sauer, P. C. Münzinger, and P. Michler, Phys. Rev. B 50, 14554 (1994).

${ }^{22}$ M. Schreck, H. Roll, J. Michler, E. Blank, and B. Stritzker, J. Appl. Phys. 88, 2456 (2000).

${ }^{23}$ E. Neu, C. Hepp, M. Hauschild, S. Gsell, M. Fischer, H. Sternschulte, D. Steinmüller-Nethl, M. Schreck, and C. Becher, New J. Phys. 15, 043005 (2013).

${ }^{24}$ E. Neu, C. Arend, E. Gross, F. Guldner, C. Hepp, D. Steinmetz, E. Zscherpel, S. Ghodbane, H. Sternschulte, D. Steinmüller-Nethl, Y. Liang, A. Krueger, and C. Becher, Appl. Phys. Lett. 98, 243107 (2011).

${ }^{25}$ B. J. M. Hausmann, B. Shields, Q. Quan, P. Maletinsky, M. McCutcheon, J. T. Choy, T. M. Babinec, A. Kubanek, A. Yacoby, M. D. Lukin, and M. Loncar, Nano Lett. 12, 1578 (2012). 